\title{
Nonlinear Analytical Model for Wire Strands
}

\author{
Chun-Lei $\mathrm{Yu}^{1}$, a, Wen-Guang Jiang ${ }^{1, \mathrm{~b}}$, Li-Juan $\mathrm{Yan}^{2, \mathrm{c}}$, \\ Jian-Ying Cui ${ }^{3, d}$, Li-Ping Zhu ${ }^{3, \text { e }}$ \\ 1School of Mechanical Engineering, Yanshan University, Qinhuangdao, Hebei, PR China \\ ${ }^{2}$ Science, Technology and Quality Department, Xuzhou Construction Machinery Group, Xuzhou, \\ Jiangsu, PR China \\ ${ }^{3}$ Technology, Research and Development Center, Juli Sling Co. Ltd, Baoding, Hebei, PR China \\ ayclfinder@163.com, bwgj@ysu.edu.cn, cyanlj@xcmg.com, dcuijy@julisling.com, \\ ezhulp@julisling.com
}

\begin{abstract}
Keywords: Wire strand; contact deformation; Hertz contact theory; tension behaviour; analytical model

Abstract. A nonlinear analytical wire strand model under axial tensile load has been developed in this paper. The model extends Costello's elastic strand model by taking into account of the effect of contact deformation between contacting wires. Hertz contact theory is adopted to establish the relationship between contact deformation and normal contact force. The tensile behaviour of a multi-layered 91-wire strand has been analysed using the model developed. The analysis results showed good agreement with the experimental data.
\end{abstract}

\section{Introduction}

Wire strands are groups of wires laid helically in successive layers over a straight center wire in a regular geometric pattern to form an integral unit to provide axial strength and stiffness. The use of these helically wound wires constitutes a wide class of vital engineering components and has played an important role in various engineering applications such as suspension bridges, sport stadium and cable-membrane structure etc.

When wire strand is loaded axially, transverse contraction will occur in the radial direction of the strand structure. The radial contraction plays an important role in determining the strand mechanical behaviour and it is mainly affected by the Poisson's ratio effect and the contact deformation between the contacting wires [1]. Owing to the complex geometry of the strand and the highly nonlinear nature of the inter-wire contacts, most analytical models to date do not take this into full consideration. Costello et al. [2] considered the Poisson's ratio effect, but neglected the contact deformation. Utting and Jones [3] evaluated the contact deformation on the basis of Hamlet's experiment. LeClair [4] studied the effect of contact deformation, which derived from Hertz contact theory, on the tension behaviour of a multilayered wire strand. But numerical inconsistencies were identified in his results [1]. Argatov [5], Gnanavel and Parthasarathy [6] had also studied the effect of contact deformation, but their works were all limited to the study of simple 7-wire strands. In this paper, a nonlinear analytical model for wire strands has been developed. It extends the Costello's elastic model by fully considering the effect of contact deformation between contacting wires in the strand.

\section{Analytical Model}

A typical geometry of wire strands analysed in this paper is shown in Fig. 1. In general, it comprises a straight core wire surrounded by several successive layers of round helical wires. The radii of helical wires in the same layer are the same. The helical directions for adjacent layers of wires are opposite. The contact between the core wire (defined as the first layer) and second-layer wires is line contact [7]. The geometric arrangement forms regular pattern layout of trellis point contacts between the rest adjacent layers of wires, which is called point contact thereafter. For an arbitrary wire in the layer $i$ $(i=1,2, \cdots, n)$, the radius is $R_{i}$, the helical angle is $\alpha_{i}$, where $n$ is the total number of wire layers in the 
strand. The strand is loaded axially with an axial force, $F$, and an axial twist moment, $M$, which represents the most basic loading case for engineering applications.

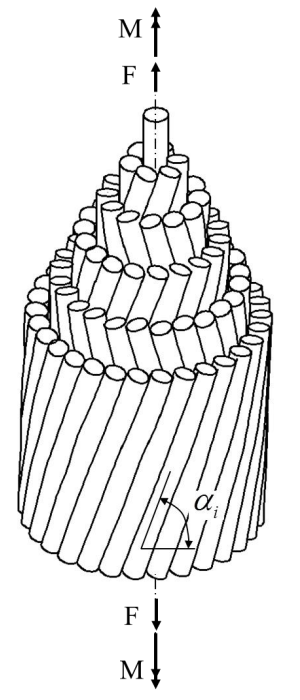

Fig. 1 Geometry of the wire strand

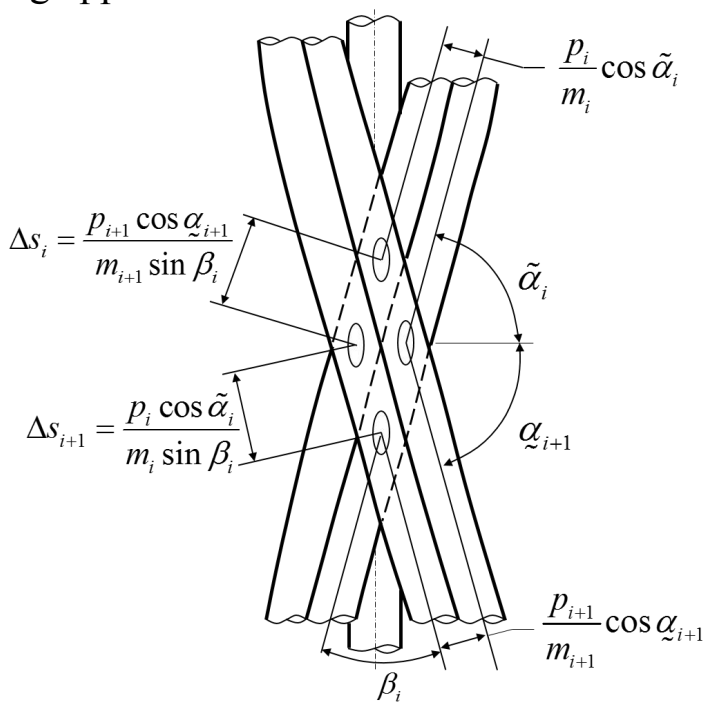

Fig. 2 Contact geometry for point contacts

It is assumed that the helical wires will deform into new helix under axial load. For an arbitrary helical wire in the $i$ th layer, the strand axial strain, $\varepsilon$, and twist rate, $\tau_{s}$, are [2]

$$
\begin{gathered}
\varepsilon=\varepsilon_{1}=\left(1+\varepsilon_{i}\right) \frac{\sin \bar{\alpha}_{i}}{\sin \alpha_{i}}-1 \\
\tau_{s}=\frac{1}{\bar{r}_{i}} \frac{1+\varepsilon}{\tan \bar{\alpha}_{i}}-\frac{1}{r_{i} \tan \alpha_{i}}
\end{gathered}
$$

where $\varepsilon_{1}$ is the axial strain of the center wire, $\varepsilon_{i}$ is the axial strain of the helical wire, $\alpha_{i}$ is the helical angle, $\bar{\alpha}_{i}=\alpha_{i}+\Delta \alpha_{i}$ is the helical angle in the loaded state, $r_{i}$ is the helix radius, $\bar{r}_{i}$ is the helix radius in the loaded state. Subscript $i(i=2,3, \cdots, n)$ represents quantities pertaining to the $i$ th layer.

The helix radius in the unloaded state, $r_{i}$, is

$$
r_{i}=R_{1}+2 \sum_{j=2}^{i-1} R_{j}+R_{i}
$$

Considering Poisson's ratio, $\mu$, and contact deformation, $\delta$, the helix radius, $r_{i}$, becomes

$$
\overline{r_{i}}=R_{1}\left(1-\mu \varepsilon_{1}\right)+2 \sum_{j=2}^{i-1}\left[R_{j}\left(1-\mu \varepsilon_{j}\right)\right]+R_{i}\left(1-\mu \varepsilon_{i}\right)-\delta
$$

where $\delta$ is caused by inter-wire contact and it can be calculated as

$$
\delta=\delta_{1}+\sum_{i=2}^{n-1} \delta_{i}
$$

and $\delta_{1}$ is the approaching distance between the core wire and its adjacent second-layer wires due to the line contact. And $\delta_{i}$ is the approaching distance between the $i$ th layer helical wires and their adjacent $(i+1)$ th layer wires at the location of the trellis crossing contact points.

According to Hertz contact theory [8], $\delta_{1}$ can be expressed as

$$
\delta_{1}=\frac{2 P_{1}\left(1-\mu^{2}\right)}{\pi E}\left[\ln \left(\frac{4 \rho_{1}}{c}\right)+\ln \left(\frac{4 \rho_{2}}{c}\right)-1\right]
$$

where $P_{1}$ is the contact force per unit length of the contact line, $E$ is the elastic modulus, $c=\sqrt{\left[8 P_{1} \rho_{1} \rho_{2}\left(1-\mu^{2}\right)\right] /\left[\pi E\left(\rho_{1}+\rho_{2}\right)\right]}$ is the half width of contact region, $\rho_{1}$ and $\rho_{2}$ are curvature radii for the core wire and the second-layer wires at contact position. 


$$
\rho_{1}=R_{1} / \sin ^{2} \underset{\sim}{\alpha}, \rho_{2}=R_{2} / \cos ^{2}\left(\underset{\sim}{\alpha}-\alpha_{2}\right)
$$

and $\underset{\sim}{\alpha}=\arctan \left(p_{2} / 2 \pi R_{1}\right)$ is the helix angle of the contact line on a wire in the second-layer.

Similarly, based on Hertz contact theory [8], $\delta_{i}$ can be expressed as

$$
\begin{aligned}
& \delta_{i}=\left[\frac{9 k^{2} P_{i}^{2}\left(1-\mu^{2}\right)^{2}}{\pi^{2} E^{2}(A+B) E\left(k^{\prime}\right)}\right]^{1 / 3} K\left(k^{\prime}\right) \\
& A=\frac{1}{4}\left(\frac{1}{\rho_{i}}+\frac{1}{\rho_{i}^{\prime}}+\frac{1}{\rho_{i+1}}+\frac{1}{\rho_{i+1}^{\prime}}\right)-\frac{1}{4}\left[\left(\frac{1}{\rho_{i}}-\frac{1}{\rho_{i}^{\prime}}+\frac{1}{\rho_{i+1}}-\frac{1}{\rho_{i+1}^{\prime}}\right)^{2}-4\left(\frac{1}{\rho_{i}}-\frac{1}{\rho_{i}^{\prime}}\right)\left(\frac{1}{\rho_{i+1}}-\frac{1}{\rho_{i+1}^{\prime}}\right) \sin ^{2} \varphi_{i}\right]^{1 / 2} \\
& B=\frac{1}{4}\left(\frac{1}{\rho_{i}}+\frac{1}{\rho_{i}^{\prime}}+\frac{1}{\rho_{i+1}}+\frac{1}{\rho_{i+1}^{\prime}}\right)+\frac{1}{4}\left[\left(\frac{1}{\rho_{i}}-\frac{1}{\rho_{i}^{\prime}}+\frac{1}{\rho_{i+1}}-\frac{1}{\rho_{i+1}^{\prime}}\right)^{2}-4\left(\frac{1}{\rho_{i}}-\frac{1}{\rho_{i}^{\prime}}\right)\left(\frac{1}{\rho_{i+1}}-\frac{1}{\rho_{i+1}^{\prime}}\right) \sin ^{2} \varphi_{i}\right]^{1 / 2}
\end{aligned}
$$

where $P_{i}$ is the normal contact force acting on the contact point between wires in the layer $i$ and $i+1$, $K\left(k^{\prime}\right)$ and $E\left(k^{\prime}\right)$ are the first and second kind of complete elliptic integrals, $k^{\prime}=\left(1-k^{2}\right)^{1 / 2}, k=b / a$, where $a$ and $b$ are the semi-major and semi-minor of the elliptic contact region, $\varphi_{i}$ is the crossing angle of the contacting wires, $\rho_{i}, \rho_{i}^{\prime}, \rho_{i+1}$ and $\rho_{i+1}^{\prime}$ are the curvature radii of the contacting wires at the contact point.

$$
\rho_{i}=R_{i}, \rho_{i}^{\prime}=\frac{r_{i}+R_{i}}{\cos ^{2} \tilde{\alpha}_{i}}, \rho_{i+1}=R_{i+1}, \rho_{i+1}^{\prime}=-\frac{r_{i+1}-R_{i+1}}{\cos ^{2}{\underset{\sim}{i+1}}_{i+1}}
$$

and $\tilde{\alpha}_{i}=\arctan \left[p_{i} / 2 \pi\left(r_{i}+R_{i}\right)\right],{\underset{\sim}{\alpha+1}}_{i+1}=\arctan \left[p_{i+1} / 2 \pi\left(r_{i+1}-R_{i+1}\right)\right], p_{i}$ and $p_{i+1}$ are the pitch lengths.

According to strand geometry, the contact force exerted by the $n$th layer on the $(n-1)$ th layer at the contact point is

$$
f_{n, n-1}=C_{n} X_{n} \Delta s_{n}
$$

where $C_{n}$ is the coefficient considering the actual contact position, $X_{n}$ is the contact force per unit length of the centerline of the $n$ th-layer wire, $\Delta s_{n}$ is the spacing between two contact points as shown in Fig. 2.

Based on Newton's third law, the contact force acting between arbitrary two contacting wire layers $i$ and $i+1$ meets

$$
f_{i, i+1}=-f_{i+1, i}
$$

where $f_{i, i+1}=C_{i} X_{i} \Delta s_{i}, f_{i+1, i}=C_{i+1} X_{i+1} \Delta s_{i+1}$.

Using the Eqs. (12) and (13), the contact force exerted by the $(i+1)$ th layer on the $i$ th layer at the contact point is the sum of the contact force from the $(i+1)$ th layer to the $n$th layer and it can be written as

$$
f_{i+1, i}=C_{i+1}\left[X_{i+1}+\sum_{j=i+2}^{n} \frac{m_{j} p_{i+1} \cos \tilde{\alpha}_{i+1}}{m_{i+1} p_{j} \cos {\underset{\sim}{j}}_{j}}\left(\prod_{k=i+1}^{j-1} \frac{\cos \tilde{\alpha}_{k}}{\cos {\underset{\sim}{k}}_{k}}\right) X_{j}\right] \Delta s_{i+1}
$$

where in above equations, $\Delta s_{i}=p_{i+1} \cos \alpha_{i+1} / m_{i+1} \beta_{i}, \Delta s_{i+1}=p_{i} \cos \tilde{\alpha}_{i} / m_{i} \beta_{i}$ (Fig. 2), $m_{i}$ is the total number of wires in the $i$ th layer, $C_{i+1}=\sqrt{\left[p_{i+1}^{2}+\left(2 \pi r_{i+1}\right)^{2}\right] /\left\{p_{i+1}^{2}+\left[2 \pi\left(r_{i+1}-R_{i+1}\right)\right]^{2}\right\}}, X_{i}$ is the contact force per unit length of the wire centerline.

Based on Costello's elasticity theory [2], when wire strand deforms under axail load, $X_{i}$ is

$$
X_{i}=N_{i}^{\prime} \bar{\tau}_{i}-T_{i} \bar{\kappa}_{i}^{\prime}
$$

where $N_{i}^{\prime}=-G_{i}^{\prime} \bar{\tau}_{i}+H_{i} \bar{\kappa}_{i}^{\prime}$ is the binormal shear force, $T_{i}=E \pi R_{i}^{2} \varepsilon_{i}$ is the wire tensile force, $G_{i}^{\prime}=\frac{E \pi R_{i}^{4}}{4}\left(\bar{\kappa}_{i}^{\prime}-\kappa_{i}^{\prime}\right)$ is the binormal bending moment, $H_{i}=\frac{E \pi R_{i}^{4}}{4(1+\mu)}\left(\bar{\tau}_{i}-\tau_{i}\right)$ is the twisting moment, 
where $\kappa_{i}^{\prime}=\cos ^{2} \alpha_{i} / r_{i}, \bar{\kappa}_{i}^{\prime}=\cos ^{2} \overline{\alpha_{i}} / \overline{r_{i}}, \tau_{i}=\sin \alpha_{i} \cos \alpha_{i} / r_{i}$ and $\bar{\tau}_{i}=\sin \bar{\alpha}_{i} \cos \bar{\alpha}_{i} / \overline{r_{i}}$ are the binormal curvatures and twists per unit length of the deformed wire centerline, respectively.

For line contact, the contact force per unit length of the contact line, $f_{2,1}$, can be calculated as

$$
f_{2,1}=C_{2}\left(X_{2}+C_{1} \frac{f_{3,2}}{\Delta s_{2}}\right)
$$

Let

$$
f_{2,1}=P_{1}, f_{i+1, i}=P_{i}
$$

where $P_{1}=\frac{\pi c^{2} E\left(\rho_{1}+\rho_{2}\right)}{8 \rho_{1} \rho_{2}\left(1-\mu^{2}\right)}, P_{i}=\frac{\pi b^{3} E(A+B)}{3 k E\left(k^{\prime}\right)\left(1-\mu^{2}\right)}$.

For each layer wires, nonlinear Eqs. (1), (2) and (17) can be achieved. They are fuctions of the unknown parameters of $\varepsilon_{i}, \Delta \alpha_{i}$ and $b$ or $c$. Intergrating all the equations for each layer wire, the nonlinear analytical model for wire strand is established. The number of equations is $3(n-1)$ which is equal to the number of the unknown parameters, thus the equation can be solved. Quasi-Newton method has been used to solve the nonlinear equations in this paper.

After the nonlinear equations are solved, the strand axial load, $F$, and torque, $M$, can be calculated using

$$
\begin{aligned}
& F=\pi R_{1}^{2} E \varepsilon+\sum_{i=2}^{n} m_{i}\left(T_{i} \sin \overline{\alpha_{i}}+N_{i}^{\prime} \cos \overline{\alpha_{i}}\right) \\
& M=\frac{E \pi R_{1}^{4}}{4(1+\mu)} \tau_{s}+\sum_{i=2}^{n} \operatorname{sgn}(i) m_{i}\left(H_{i} \sin \overline{\alpha_{i}}+G_{i}^{\prime} \cos \overline{\alpha_{i}}+T_{i} \overline{r_{i}} \cos \overline{\alpha_{i}}-N_{i}^{\prime} \bar{r}_{i} \sin \overline{\alpha_{i}}\right)
\end{aligned}
$$

where if right lay, $\operatorname{sgn}(i)=+1$; if left lay, $\operatorname{sgn}(i)=-1$.

\section{Numerical Analysis}

The tensile behaviour of a six layered 91-wire strand was analysed using the model developed in this paper. Table 1 details the geometry data of the strand construction. The radius of the core wire is greater than the radii of the helical wires, which ensure that the contact occurs only between adjacent layers. The Young's modulus is $210 \mathrm{GPa}$ and Poisson's ratio is 0.3 . A strand axial strain $\varepsilon$ of 0.008 was applied in increments of 0.001 in the analysis. Non-rotation end constraint conditions are imposed.

To validate the analytical model developed, tensile test on the 91-wire strands was conducted. The experiment arrangement is shown in Fig. 3. A $5000 \mathrm{kN}$ hydraulic testing machine designed for wire rope tests was used. The length of the sample was $2800 \mathrm{~mm}$ and its ends were fixed in the conical mould using casting method and then clamped on the tension machine as shown in Fig. 3(b). Extensometer was used to measure the strand extension and mounted on the sample with an original length of $1962 \mathrm{~mm}$ as shown in Figs. 3(c) and 3(d). Vernier caliper with wide mouth plier was used to measure the strand diameter.

Table 1 The geometry data of the six layered 91-wire strand

\begin{tabular}{cccccc}
\hline $\begin{array}{c}\text { Layer } \\
\text { number } i\end{array}$ & $\begin{array}{c}\text { Number of } \\
\text { wires } m_{i}\end{array}$ & $\begin{array}{c}\text { Wire diameter } \\
2 R_{i}(\mathrm{~mm})\end{array}$ & $\begin{array}{c}\text { Pitch length } \\
p_{i}(\mathrm{~mm})\end{array}$ & $\begin{array}{c}\text { Helical angle } \\
\alpha_{i}\left(^{\circ}\right)\end{array}$ & $\begin{array}{c}\text { Lay } \\
\text { direction }\end{array}$ \\
\hline 1 & 1 & 5.00 & - & 90 & - \\
2 & 6 & 4.55 & 106 & 74.2 & $\mathrm{RH}$ \\
3 & 12 & 4.55 & 207 & 74.2 & $\mathrm{LH}$ \\
4 & 18 & 4.55 & 307 & 74.2 & $\mathrm{RH}$ \\
5 & 24 & 4.55 & 408 & 74.2 & $\mathrm{LH}$ \\
6 & 30 & 4.55 & 509 & 74.2 & $\mathrm{RH}$ \\
\hline
\end{tabular}




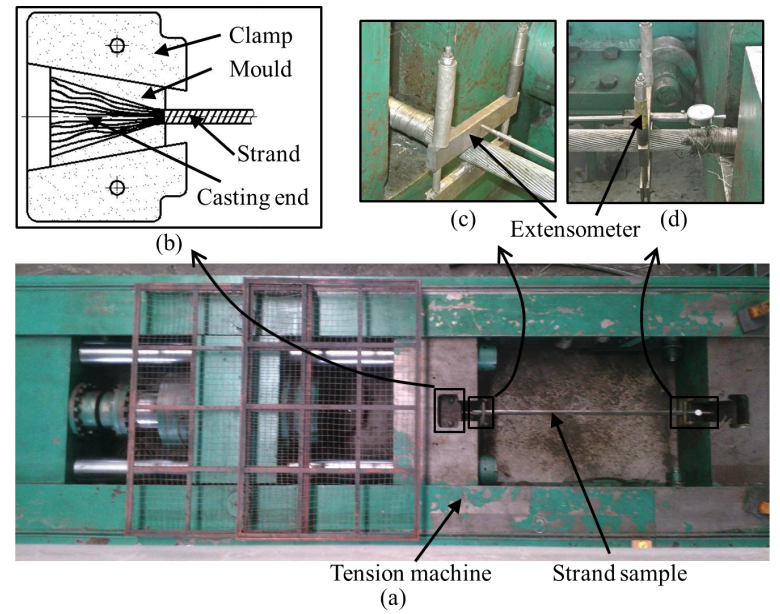

Fig. 3 Tensile experiment arrangement

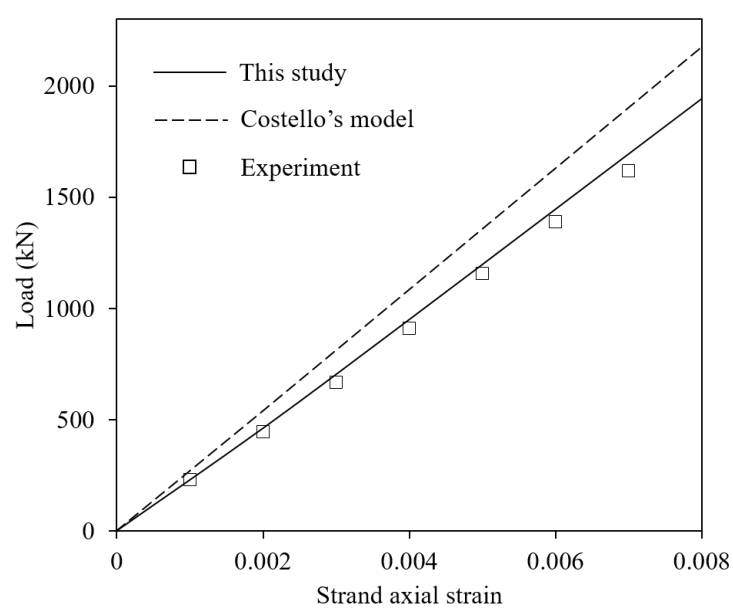

Fig. 4 Variation of load with strand axial strain

Fig. 4 shows the strand axial load as a function of the applied axial strain. The results have been compared with those obtained from experiment and Costello's elastic model. From this figure it can be seen that the present analytical model shows better agreement with the experiment, whereas Costello's model gives too high load predictions for given axial strains.

Fig. 5 shows the variation of axial stress in a single wire of each layer with strand axial strain. It can be seen that the stress level in the core wire is higher than those of the helical wires. And the stress levels for the helical wires are quite uniform during the loading process. This means that the design of this wire strand construction is rational.

Fig. 6 shows the variation of contact approaching distance between adjacent layers of contacting wires for different strand axial strain levels. From this figure it can be seen that the maximum contact deformation occurs between the second-layer wires and the third-layer wires, which is the innermost trellis crossing contact location. It can be also seen that, with increasing strand axial strain, the stiffness of the crossing trellis contact increases. This agrees with the rules of Hertz contact theory.

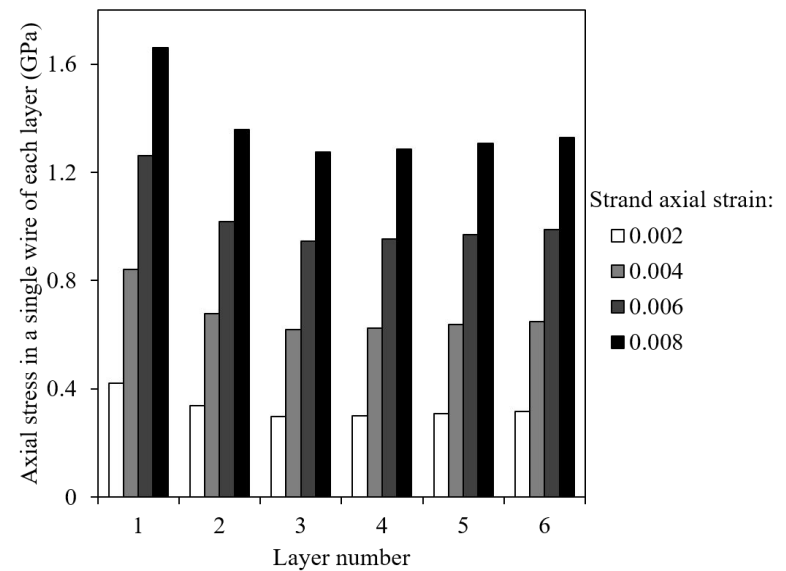

Fig. 5 Axial stress in a single wire of each layer

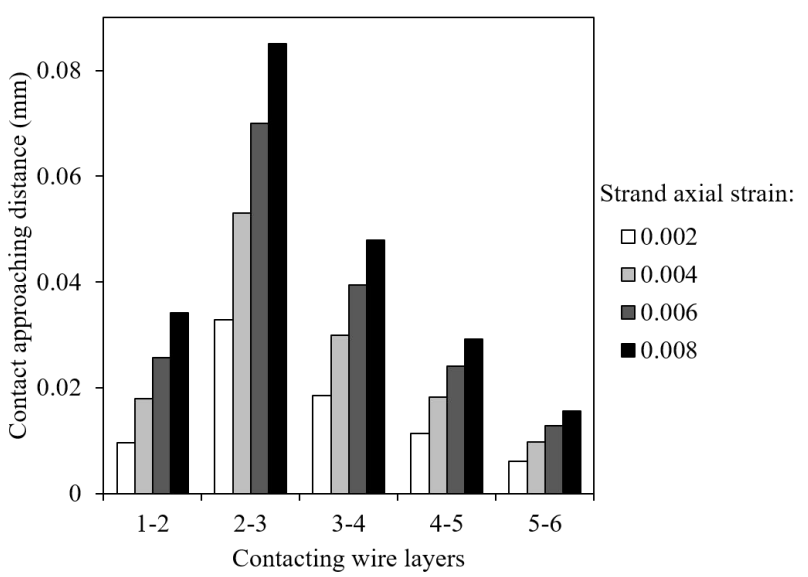

Fig. 6 Contact approaching distance

With the increase of the strand axial load, the diameter of the strand decreases. To further validate the model developed in this paper, variation of the strand diameter with applied strand axial load was experimentally measured. Table 2 shows the strand diameter changes from both the numerical models and experiments. $F_{0}=2129 \mathrm{kN}$ is the strand minimum breaking load of the six layered 91 -wire strand. The diameter of the tested strand is slightly larger than its nominal diameter due to the geometry imperfection from manufacturing process. As the amount of diameter contraction due to extension load is quite small, it would be clearer to directly show the relative changes of diameters between loading intervals. Fig. 7 compares the relative diameter changes between different loading levels from different models and experiments. From this comparison it can be seen that the new model compares well with the experimental values. This indicates that the contact deformation should be considered when developing accurate mechanical models for multi-layered strands. 
Table 2 Variation of strand diameters ( $\mathrm{mm})$

\begin{tabular}{ccccc}
\hline $\begin{array}{c}\text { Loading level } \\
\text { (extension strain) } F(\varepsilon)\end{array}$ & $\begin{array}{c}\text { Strand nominal } \\
\text { diameter }\end{array}$ & $\begin{array}{c}\text { Costello's } \\
\text { model }\end{array}$ & $\begin{array}{c}\text { Analytical model } \\
\text { (his study) }\end{array}$ & Experiment \\
\hline $0.2 F_{0}(0.00184)$ & 50.5 & 50.475 & 50.326 & 49.850 \\
$0.3 F_{0}(0.00276)$ & 50.5 & 50.462 & 50.265 & 49.775 \\
$0.4 F_{0}(0.00368)$ & 50.5 & 50.449 & 50.210 & 49.750 \\
$0.5 F_{0}(0.00460)$ & 50.5 & 50.436 & 50.166 & 49.725 \\
\hline
\end{tabular}

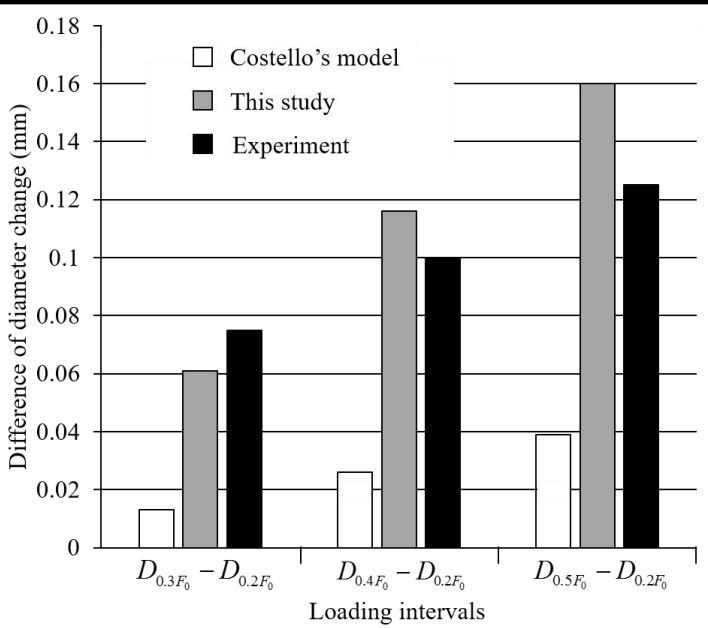

Fig.7 Comparison of relative diameter changes between different loading levels

\section{Conclusion}

A nonlinear analytical strand model which fully takes into account the contact deformation between the contacting wires has been developed in this paper. A multi-layered wire strand construction has been analysed using the model developed. For the global tensile rigidity, the nonlinear model prediction shows excellent agreement with the experimental results. The neglecting of contact deformation between contacting wires in Costello's elastic model leads to about $10 \%$ higher prediction of the axial extension rigidity for this multi-layered wire strand construction.

\section{Acknowledgements}

This work was financially supported by the Hebei Provincial Natural Science Foundation (E2015203196).

\section{References}

[1] M. Raoof, I. Kraincanic. Critical examination of various approaches used for analyzing helical cables. Journal of Strain Analysis for Engineering Design. Vol. 29 (1994), p. 43-55.

[2] G. A. Costello: Theory of wire rope, 2nd ed (Springer Verlag, New York 1997).

[3] W. S. Utting, N. Jones. The response of wire rope strands to axial tensile loads. 1. Experimental results and theoretical predictions. Journal of Mechanical Sciences. Vol. 29(1987), p. 605-619.

[4] R. A. LeClair. Axial response of multilayered strands with compliant layers. Journal of Engineering Mechanics. Vol. 117(1991), p. 2884-2902.

[5] I. Argatov. Response of a wire rope strand to axial and torsional loads: Asymptotic modeling of the effect of interwire contact deformations. International Journal of Solid and Structures. Vol. 48(2011), p. 1413-1423.

[6] B. K. Gnanavel, N. S. Parthasarathy. Effect of interfacial contact forces in single layer cable assemblies. International Journal of Mechanics \& Materials in Design. Vol. 8(2012), p. 183-195.

[7] W. G. Jiang, M. S. Yao and J. M. Walton. A concise finite element model for simple straight wire rope strand. International Journal of Mechanical Sciences. Vol. 41 (1999), p. 143-161.

[8] K. L. Johnson. Contact mechanics, 6th ed (Cambridge University Press, Cambridge 1996). 